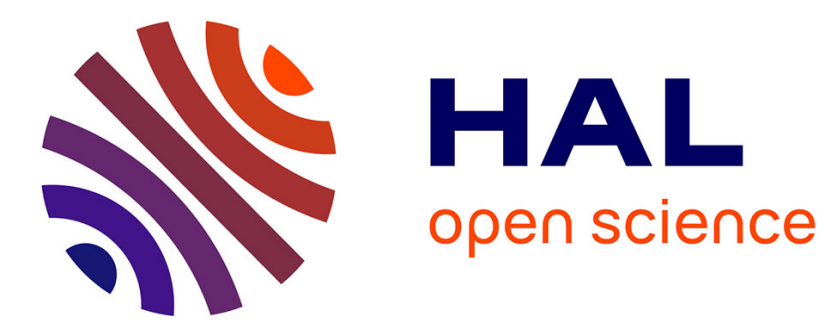

\title{
Interferences of Peltier thermal waves produced in ohmic contacts upon integrated circuits
}

W. Claeys, S. Dilhaire, V. Quintard, D. Lewis, T. Phan, J. Aucouturier

\section{To cite this version:}

W. Claeys, S. Dilhaire, V. Quintard, D. Lewis, T. Phan, et al.. Interferences of Peltier thermal waves produced in ohmic contacts upon integrated circuits. Journal de Physique IV Proceedings, 1994, 04 (C7), pp.C7-195-C7-198. 10.1051/jp4:1994747 . jpa-00253276

\section{HAL Id: jpa-00253276 https://hal.science/jpa-00253276}

Submitted on 1 Jan 1994

HAL is a multi-disciplinary open access archive for the deposit and dissemination of scientific research documents, whether they are published or not. The documents may come from teaching and research institutions in France or abroad, or from public or private research centers.
L'archive ouverte pluridisciplinaire HAL, est destinée au dépôt et à la diffusion de documents scientifiques de niveau recherche, publiés ou non, émanant des établissements d'enseignement et de recherche français ou étrangers, des laboratoires publics ou privés. 


\title{
Interferences of Peltier thermal waves produced in ohmic contacts upon integrated circuits
}

\author{
W. Claeys, S. Dilhaire, V. Quintard, D. Lewis, T. Phan and J.L. Aucouturier* \\ Centre de Physique Moléculaire Optique et Hertzienne, Université de Bordeaux I, CNRS, 351 cours de la \\ Libération, 33405 Talence cedex, France \\ * Laboratoire de Microélectronique (IXL), Université de Bordeaux I, CNRS, 351 cours de la Libération, \\ 33405 Talence cedex, France
}

\begin{abstract}
We have experimentally detected a Peltier effect produced by the current flow « ohmic contacts upon integrated circuits. These contacts are small size heat sources which are very useful for investigating integrated circuits. We have characterised their thermal properties with a high resolution interferometric laser probe by measuring absolute thermal expansions. Subpicometric resolution has allowed original imaging of semiconductor components : we have mapped the thermal waves created at ohmic contacts under normal operating conditions.
\end{abstract}

\section{INTRODUCTION}

Thermoelectrical effects, like Joule, Peltier, Thomson and Seebeck occur in semiconductor devices. These effects have been studied experimentally before $[1,2]$. We have developed an appropriate instrumentation for the thermal characterisation of semiconductor devices [3,4] at a microelectronic scale and have undertaken systematic studies of thermoelectric effects in integrated circuits.

In the present work we show metal semiconductor contacts to be the source of a Peltier thermal wave. Such original heat sources can be very useful for thermal probing inside semiconductor devices.

In integrated circuits, links between the power supply and semiconductor integrated components are realised through ohmic contacts. An ohmic contact is defined as a metal-semiconductor connection that has negligible resistance relative to the bulk or spreading resistance of the semiconductor. We have investigated the thermal effects of current in $\mathrm{Al}-\mathrm{Si}$ contacts and have observed, besides the Joule heating ( $\mathrm{I}^{2}$ dependent), an important Peltier heating process (I dependent) located at the metal-semiconductor interface.

The Peltier heat source in ohmic contacts is a very interesting one in terms of thermal wave probing : it is of very small size (located within a $\mu \mathrm{m}^{3}$ volume), it is very easy to modulate and it is part of all components in integrated circuits. It is worth to note that for sine wave current excitation, there is no average temperature change due to the Peltier effect. Furthermore, there are no electron-hole pairs formed like in conventional photothermal analysis of semiconductors.

We have followed the propagation - amplitude and phase - of low frequency surface waves in a doped semiconductor, resulting from thermal wave propagation. We have also studied interferences from two thermal waves originated at ohmic contacts linked together by a semiconductor resistance where a sine wave current is flown. The Peltier heat sources at the two ohmic contacts, have opposite phases and the associated thermal waves will interfere destructively at mid distance between the sources. We also show an example of Peltier thermal wave imaging of a semiconductor component. The image is obtained by mapping the thermal wave amplitude propagating from the ohmic contacts of the functioning component. This imaging technique shows to be interesting for semiconductor homogeneity and reliability testing.

We have studied these surface waves with a high resolution stabilised Michelson interferometer we have built [3]. We have observed with this probe, absolute surface normal displacements amplitudes 
of a few $10^{-15} \mathrm{~m}$ upon silicon circuits for sine wave current excitation. In our approach, the component has its normal electrical excitation and the resulting thermal behaviour is detected in synchronism with the electrical source.

\section{HIGH RESOLUTION INTERFEROMETRIC LASER PROBE}

We have built and developed a Michelson type interferometer designed for the analysis of semiconductor components [1]. Three main requirements have to be met: to visualise the point under test upon the device with micrometric resolution, to cope with the different reflectances of the materials involved in electronic components $(\mathrm{Al}, \mathrm{Si}, \ldots)$ and finally to detect the interferometer response in synchronism with the circuit.

Figure 1 shows a schematic view of the interferometric laser probe, a homodyne stabilised Michelson interferometer. The laser is a stabilised polarised HeNe laser . The beam splitting element of the interferometer is a polarising prism. By rotating a half wave plate $(\lambda / 2)$ it is possible to partition the intensity of the reference arm to that of the probe arm in order to equalise the reflected intensities and to obtain high contrast interference fringes. In the reference and probe arm a quarter wave plate ( $\lambda / 4)$ is inserted. The linear polarisation of the incoming light is rotated by $90^{\circ}$ when coming out, as it has passed twice the plate. This allows to reflect all the intensity of the returning beams to the photodetector and the polarising prism acts as an optical insulator. The two beams have orthogonal linear polarisations. To obtain interferences, both polarisations are projected at $45^{\circ}$ upon a same axis by passing through a polarising beam splitter (prism) before the photodetector. A microscope objective focuses the probe beam upon the surface of the component under test. The phase of the reflected beam is modulated by the surface normal displacements. The sample is mounted on a 3-D micrometric translation stage.

The laser impact upon the sample can be viewed on a CCD camera by moving the mirror in front of the reflected probe beam and by reducing the laser beam intensity with an attenuator. The lateral resolution is $1 \mu \mathrm{m}$. For small surface displacements $(<\lambda / 20, \lambda=6328 \AA)$, the interferometer is actively stabilised.

The signal is measured in synchronism with the excitation signal and recorded by a digital oscilloscope or a lock-in amplifier.

Absolute values of the surface displacement are obtained from comparison of the photodetected signal amplitude with the fringe amplitude obtained by moving the piezomirror in the reference arm.

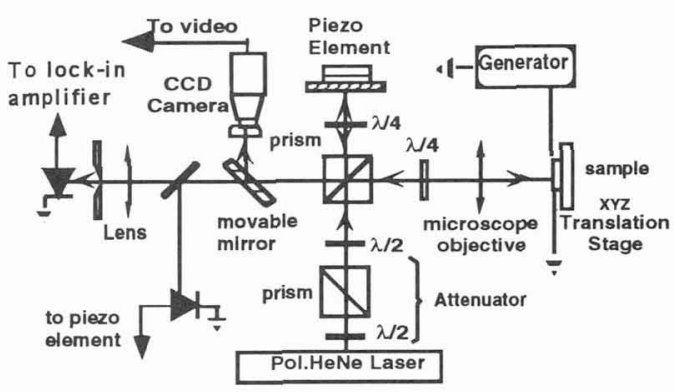

figure 1: the interferometer

\section{TEST STRUCTURE}

We have chosen to study the thermal response of a very simple structure upon an integrated circuit : a semiconductor surface resistor. This is found in a Van der Pauw test structure (figure 2).

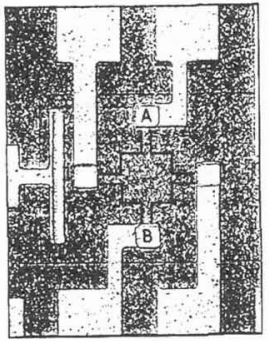

figure 2a: Van der Pauw test structure

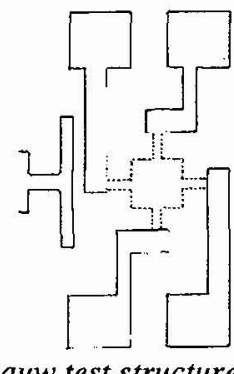

These are designed upon experimental integrated circuits in order to measure with a four points method the conductivity of the doped semiconductor material. Figure 2a shows a picture of the test structure together with a sketch of the metal lines (full lines) and resistor limits(dotted lines). Figure $2 \mathrm{~b}$ shows a 
schematic view of the structure : the contour shows the limits of the mask used for the diffusion of the impurities and the zoom shows the dimensions of the resistive structure in its narrow part. Four ohmic contacts are connected at the extremities of the diffused cross. We use the structure as a surface resistor by flowing current through two opposite ohmic contacts (A and B in figure 2a).

\section{THERMOELECTRICAL EFFECT IN TEST STRUCTURE}

The main thermoelectric effect produced by driving a current from contact $A$ to $B$ (fig $2 b$ ) through the semiconductor resistor is the Joule effect in the contact and surface resistors. The corresponding thermal behaviour of the structure has been presented in earlier work $[3,4]$.

A Peltier thermoelectric effect is located at the ohmic contacts when current flows. Figure 3 shows energy band diagrams of a metal-semiconductor structure typical of Al-Si interfaces. A satisfactory ohmic contact supplies the required current with a voltage drop that is sufficiently small compared to the drop across the active region of the device. Two contributions allow the current transport across the contact, the thermionic emission current (fig 3a) (mainly for low doping concentration) and tunnelling (fig 3b) (for high doping concentration). For both current transport mechanisms across the contact, it is clear that electrons undergo a small potential jump $\left(\mathrm{E}_{\mathrm{C}}-\mathrm{E}_{\mathrm{F}}\right)$ at the junction. This produces a very local thermoelectric effect, which is current dependent, the Peltier effect. For sine wave current excitation, this effect is easily distinguished from the Joule effect as it has a first harmonic response while the latter has a second harmonic response.

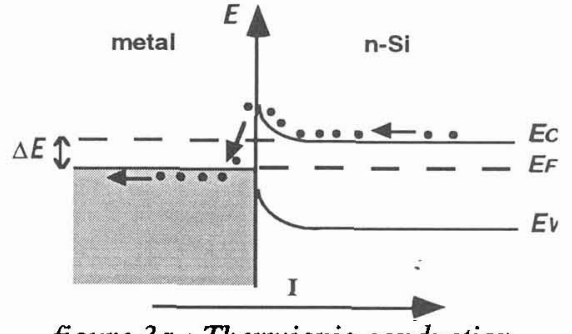

figure $3 a$ : Thermionic conduction

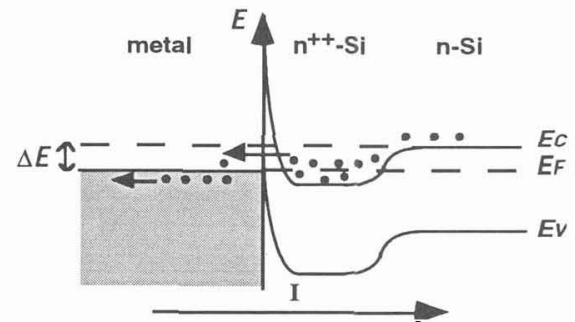

figure $3 b:$ Tunnel effect conduction

\section{RESULTS}

\subsection{Joule and Peltier thermal expansion at ohmic contact}

Figure 4 shows the current dependence of the first and second harmonic surface displacement amplitudes resulting from a pure sine wave current excitation at $20 \mathrm{kHz}$. They correspond respectively to Peltier and Joule heating of the ohmic contact. Pure linear and quadratic responses are observed.

\subsection{Interferences of thermoelastic surface waves}

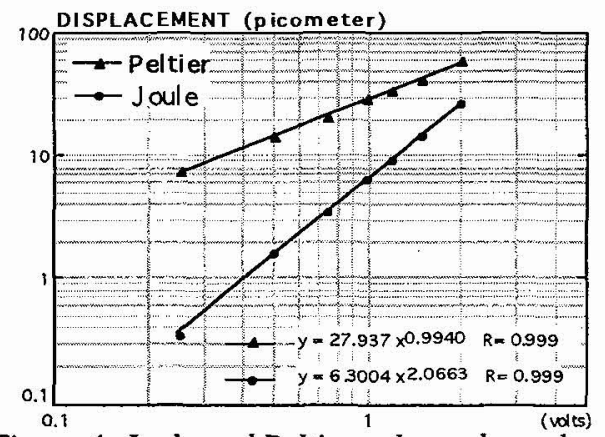

Figure 4: Joule and Peltier voltage dependence

Figure 5 a shows the amplitude distribution of first harmonic thermoelastic surface waves along the $112 \mu \mathrm{m}$ line of semiconductor resistor joining the two ohmic contacts (A, B in figure 2a). Both contacts are Peltier heat sources of opposite phase generating thermal waves in the semiconductor bulk. A simple exponential decay starting from the sources is observed, except in the central region where destructive interferences of the two surface waves take place. The full line in fig $5 \mathrm{a}$ was obtained from a simple simulation of surface waves originated from two point sources. These waves undergo exponential absorption and linear dephasing while propagating. Fig 5 b shows the phase measurements and simulation for the same situation. Interferences are also clearly observed around the $180^{\circ}$ phase jump at midway. 
Simple exponential amplitude and linear phase decays are observed in all directions near the ohmic contact sources.

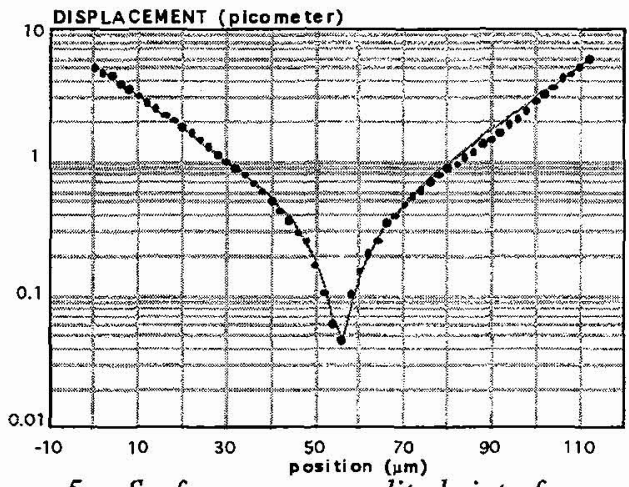

figure 5.a: Surface waves amplitude interferences

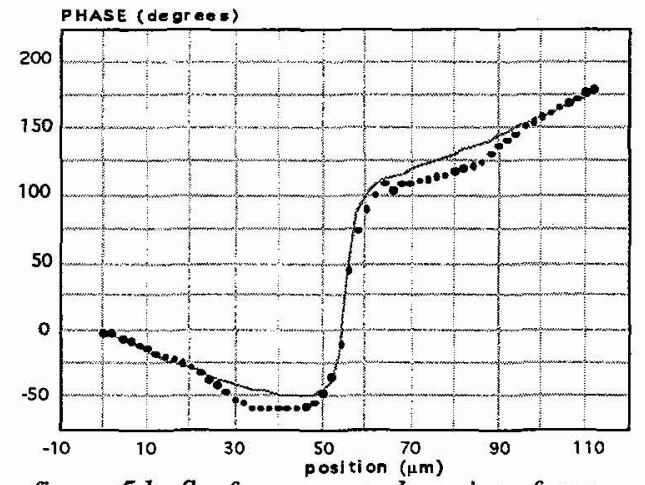

figure 5.b: Surface waves phase interferences

\subsection{Component imaging from Peltier thermal wave propagation upon integrated circuit}

By flowing a sine wave current through opposite contacts in the Van der Pauw test structure presented in figure 2 , we generate thermoelastic surface waves in the entire component. Figure 6 presents an image of the component obtained by mapping the amplitude of the thermoelastic first harmonic surface displacement.

We observe a monotonous decrease in amplitude starting from both ohmic contacts. This gives information concerning the homogeneity of the semiconductor material around the contacts.

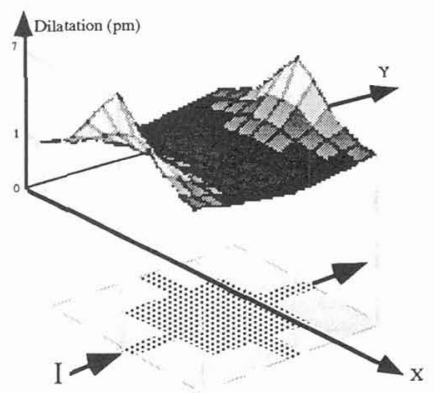

figure 6: Peltier displacement imaging of IC

\section{CONCLUSION}

We have proposed an original method to generate thermal waves from ohmic contacts in integrated circuits. The production of such waves in a normal functioning component produces very stable and reliable generation conditions which allows a high resolution analysis. In this work all the data deal with picometer surface displacements.

Further work will focus upon the possibility to derive pertinent informations on the properties of the semiconductor material. Applications in the field of quality and reliability testing of semiconductor devices are readily seen, like functioning tests, current sensing, homogeneity, etc...

\section{References}

[1] Quantitative separation of mechanisms for power dissipation in solar cells by photoacoustic and photovoltaic measurements. Harvey Flaisher, Martin Wolf and David Cahen. J. Appl. Phys. 66 (4) 15 August 1989

[2] Electrothermal measurements: A calorimetric method to examine power dissipation in photovoltaic devices. Bettine Büchner, Harvey Flaisher, Martin Wolf and David Cahen. J. Appl. Phys. 67 (9) 1 May 1990

[3] Laser beam probing of thermal behaviour of electronic components and its application in quality and reliability testing. W. Claeys, S. Dilhaire and V. Quintard -Microelectronic Engineering 24, (1994), p 411-420.

[4] Thermoreflectance optical test probe for the measurement of current-induced temperature changes in microelectronic components. W. Claeys, S. Dilhaire, V. Quintard, J.P. Dom and Y. Danto. Quality and Reliability Engineering International, Vol.9, 303-308, (1993) . 